\title{
Re-evaluation of an Optimized Second Order Backward Difference (BDF2OPT) Scheme for Unsteady Flow Applications
}

\author{
Veer N. Vatsa* \\ Mark H. Carpenter ${ }^{\dagger}$ \\ David P. Lockard ${ }^{\ddagger}$ \\ NASA Langley Research Center, Hampton,VA 23681
}

\begin{tabular}{llll}
\multicolumn{3}{c}{ Nomenclature } \\
$a$ & speed of sound & Greek: \\
$C_{p}$ & $=\frac{2\left(p-p_{\infty}\right)}{\rho_{\infty} U_{\infty}^{2}}$, surface pressure coefficient & $\rho$ & fluid density \\
$D$ & cylinder diameter & $\theta$ & azimuthal angle $(x-y$ plane $)$ \\
$i$ & $=\sqrt{-1}$, imaginary number & $\nu$ & kinematic viscosity \\
$M$ & Mach number & Superscript: \\
$p$ & pressure &,$\quad$ perturbation quantity $\left(\right.$ e.g. $\left.\rho^{\prime}=\rho-\rho_{\infty}\right)$ \\
$R e$ & $=\frac{|\mathbf{V}| D}{\nu}$, Reynolds number & Subscript: \\
$u, v, w$ & Cartesian fluid velocity components & $\infty$ & freestream quantity \\
$u_{*}$ & friction velocity & $r m s \quad$ root mean square \\
$\mathbf{V}$ & Cartesian velocity vector & & \\
$x, y, z$ & Cartesian coordinates & & \\
$y^{+}$ & $=\frac{u_{*} y}{\nu}$, nondimensional wall distance & &
\end{tabular}

Recent experience in the application of an optimized, second-order, backward-difference (BDF2OPT) temporal scheme is reported. The primary focus of the work is on obtaining accurate solutions of the unsteady Reynolds-averaged Navier-Stokes equations over long periods of time for aerodynamic problems of interest. The baseline flow solver under consideration uses a particular BDF2OPT temporal scheme with a dual-timestepping algorithm for advancing the flow solutions in time. Numerical difficulties are encountered with this scheme when the flow code is run for a large number of time steps, a behavior not seen with the standard secondorder, backward-difference, temporal scheme. Based on a stability analysis, slight modifications to the BDF2OPT scheme are suggested. The performance and accuracy of this modified scheme is assessed by comparing the computational results with other numerical schemes and experimental data.

\section{Introduction}

Significant progress has been made in the field of computational fluid dynamics (CFD) during the last three decades, and modern CFD codes are applied routinely for solving steady, viscous flows on complex configurations using the Euler and Reynolds averaged Navier-Stokes equations. ${ }^{1-6}$ However, the computational methodology is not as mature for the solution of unsteady flows, which is one of the next big challenges for the CFD community. The unsteadiness could be self-induced, such as for large separated flows, be created by flow control devices, or generated by many other mechanisms. Obtaining statistically meaningful solutions for such flow problems requires significant computational resources. However, advances in multi-core, multi-processor computer technology and numerical algorithms that take advantage of massive parallelization have resulted in significant reduction in wall clock time to obtain statistically converged solutions for problems of practical interest.

An interesting and pertinent application of unsteady flow simulations is to provide input data to acoustic noise propagation codes. Such applications frequently have significant fluctuations over a range of frequencies and require unsteady solutions over a very large number of time steps to produce broadband statistical data with adequate temporal resolution. However, care must be exercised in selecting the numerical scheme for such applications because long time

\footnotetext{
* Senior Research Scientist, CASB, RTD, Associate Fellow AIAA

$\dagger$ Senior Research Scientist, CASB, RTD

${ }^{\ddagger}$ Senior Research Scientist, CASB, RTD, Senior Member AIAA

This material is declared a work of the U.S. Government and is not subject to copyright protection in the United States.
} 
computations allow minute numerical instabilities (e.g. unstable eigenvalues of magnitude $10^{-5}$ ) to grow without bound, a phenomena that might remain unnoticed for short time computations.

One possibility of improving the numerical efficiency is by adopting a higher-order, multi-stage, temporal scheme (e.g. 3rd- or 4th-order, implicit, Runge-Kutta ${ }^{7}$ ). Indeed, the efficacy of high-order schemes has been demonstrated by several researchers on problems with varied error tolerances, particularly those that require high levels of accuracy. ${ }^{8-10}$ Adopting high-order schemes in large-scale engineering applications is not without complications. High-order schemes place an additional burden on the numerical algorithm used to solve the nonlinear set of equations, and only realize their full potential when the solver delivers a convergence rate that is relatively independent of time-step. Time-step independent convergence rates may be a difficult goal to achieve in general. Solver technology is one of the least mature disciplines among the many that are needed in the CFD community, and is presently the focus of extensive research. Placing any additional burden on the solver is not to be taken lightly. Hence, despite the recent successes of high-order schemes, there is still the need for reliable and robust, second-order methods.

Herein, we focus strictly on multistep methods; in particular, the backward-differentiation formula (BDF) class of multistep schemes. These schemes have historically been very popular for large scale scientific computations because of their simplicity, nonlinear robustness, and relative efficiency. Perhaps the most popular of the BDF class of schemes is the second-order scheme that requires three time levels (BDF2). In addition to being second order accurate, it can be used for arbitrarily large time-steps ( $A$-stability) without encountering numerical instability. Our ultimate goal in this work is to identify the most accurate BDF2 scheme that is stable for arbitrarily large time-steps. We identify an alternative second-order BDF2 scheme, (one of a class of optimized, second-order, backward difference methods referred to as "BDF2OPT schemes"), with an error constant half as large as the conventional BDF2 scheme. The new scheme is extensively tested on model problems as well as a realistic problem involving the unsteady flow past a circular cylinder.

\section{Governing Equations and Numerical Scheme}

For the present work, the Reynolds-averaged Navier-Stokes equations are used as the governing equations to model the flow field. The discrete form of these equations on unstructured grids is solved using the Computational Fluid Dynamics (CFD) code known as FUN3D developed originally by Anderson and Bonhaus. ${ }^{11}$ The FUN3D code has gone through significant modifications over the years by a team of researchers at NASA Langley using modern software practices. ${ }^{12}$ Solutions are obtained either in a time-accurate manner with a constant time step at every grid point or with variable time stepping to accelerate convergence to a steady state. The effects of turbulence are modeled by using either the Spalart-Allmaras (SA) ${ }^{13}$ model or with Menter's two-equation shear stress transport (SST) ${ }^{14}$ model.

FUN3D has a wide range of flux schemes and flux limiters from which to choose. For the current work, Roe's fluxdifference splitting scheme ${ }^{15}$ is used without a flux limiter. For steady flows, the solution at each time step is updated with a backwards Euler time-differencing scheme and the use of local time stepping. At each time step, the linear system of equations is relaxed in a red-black fashion with a point implicit procedure. ${ }^{16}$ For unsteady flow problems, a dual-time-stepping scheme ${ }^{17}$ is employed. A variety of time marching schemes are available in FUN3D, including a second-order, backward-differencing formulation (BDF2), and an optimized, second-order, backward-differencing formulation (BDF2OPT). These will be discussed further in the next section.

\section{Temporal Discretization}

We focus strictly on multistep methods in this study; in particular, we examine the backward-differentiation formula (BDF) class of multistep schemes. These schemes, popularized by Gear, ${ }^{18}$ are widely used for the integration of differential equations. The BDF schemes applied to the differential equation $u_{t}=f(u, t)$, can be expressed in the form

$$
\sum_{j=0}^{k} \delta_{j} \nabla^{j} u_{n+1}=\Delta t f_{n+1} .
$$

In these equations, $\nabla^{j}$ represents the backward difference operator and $\Delta t$ represents the time step. The values of the coefficients $\delta_{j}$ are easily derivable from a Taylor series expansion and are reported in Ref. 19 (pp. $365-366$ ).

It is well known that only the first- and second-order BDF schemes (BDF1 and BDF2) are $A$-stable, while BDF schemes of order three and higher are only $A(\alpha)$-stable. Dahlquist's famous theorem ${ }^{20}$ states that

Theorem 1 Let p represent the polynomial order of the temporal discretization. An A-stable multistep method must be 
of order $p \leq 2$. Furthermore, if the order is 2 , then the error constant satisfies

$$
C \leq-\frac{1}{12}
$$

Here, $C$ is the coefficient of the leading truncation error term.

$A$-stability requires that all points in the left half of the complex plane (LHP), including the imaginary axis, are damped over one time step. $A(\alpha)$-stability allows for growth in a wedge region of the included angle, $(90-\alpha)$, that lies in the LHP between the imaginary axis and the line joining the origin and the tangency point of the root locus of the stability curve. More detailed discussion on $A(\alpha)$-stability is available in Refs. 20 and 21.

This second barrier of Dahlquist limits the utility of the BDF class of schemes for $p \geq 3$ as a general purpose scheme. A noteworthy exception is the case $p=3$. For example, Melson et al. ${ }^{22}$ showed that the BDF3 scheme was viable for many CFD applications. However, numerical instabilities were encountered by Vatsa and Carpenter ${ }^{23}$ when using the BDF3 scheme to simulate the unsteady flow created by synthetic jets, thus underscoring the pitfalls of using a conditionally stable scheme.

The stability characteristics for various BDF schemes are given in Table 1.

\begin{tabular}{|c|c|c|c|}
\hline Method & Levels & Order & $A(\alpha)$ \\
\hline BDF1 & 2 & 1 & $A\left(90^{\circ}\right)$ \\
BDF2 & 3 & 2 & $A\left(90^{\circ}\right)$ \\
BDF3 & 4 & 3 & $A\left(86.03^{\circ}\right)$ \\
BDF4 & 5 & 4 & $A\left(73.35^{\circ}\right)$ \\
\hline
\end{tabular}

Table 1. Properties of BDF methods.

Given the "impossibility" of constructing $A$-stable multistep BDF methods higher than second order (of any number of levels), an obvious related question is "What is the most accurate $A$-stable BDF scheme that can be constructed?" Or more precisely, "Given an arbitrary number of time levels, what is the $A$-stable BDF scheme with a leading order truncation error closest to the $C \leq-\frac{1}{12}$ barrier?" Asymptotically, one could imagine approaching the barrier as the number of time levels is increased. Clearly, large-scale computations place practical limitations (storage and work) on the required number of time-levels. Furthermore, preliminary investigation suggests that most benefits are achieved by including one or two additional time levels. Thus, in this work we focus exclusively on the four- and five-time-level, two parameter family of BDF schemes constructed from a linear combination of the BDF2, BDF3 and BDF4 schemes.

We now begin by re-optimizing the BDF2 scheme subject to the $A$-stability constraint and four-time-levels. ${ }^{\S}$ A one parameter family of four-time-level, second-order, multistep schemes [BDF2OPT $(\beta)]$, is achieved by using a linear combination of the BDF2 and BDF3 schemes:

$$
\operatorname{BDF} 2 \mathrm{OPT}(\beta)=(\beta) \operatorname{BDF} 3+(1-\beta) \operatorname{BDF} 2
$$

Next, we adjust the parameter $\beta$ to minimize the leading order truncation error of the $\operatorname{BDF} 2 \mathrm{OPT}(\beta)$ scheme, subject to the constraint that the resulting scheme is $A$-stable. Note that adjusting the parameter $\beta$ merely scales the coefficient of the leading order truncation term, $C=\frac{-4}{12}$, of the original BDF2 scheme by the factor $(1-\beta)$. Thus, based on Dahlquist's theorem, we should test the schemes on the interval $0 \leq \beta \leq \frac{3}{4}$.

The stability properties of multistep schemes are determined by assessing where in the complex plane the roots of the temporal polynomial lie. The root locus curves of the BDF class of schemes is given by

$$
\mu=\sum_{j=1}^{k} \frac{1}{j}[1-\exp (-i \theta)]^{j}
$$

For example, given the BDF2 scheme $(k=2)$, the real part of the root locus curve is defined by $\operatorname{Re}(\mu)=\frac{3}{2}-$ $2 \cos (\theta)+\frac{1}{2} \cos (2 \theta)$, which upon inspection reveals that $\operatorname{Re}(\mu) \geq 0$ because the root locus curve never crosses the imaginary axis. Thus, the scheme is $A$-stable because all LHP eigenvalues are damped.

Combining Eqns. (1) and (2) yields an equation for the root locus of the $\operatorname{BDF} 2 \mathrm{OPT}(\beta)$ scheme:

$$
\begin{aligned}
\mu & =-\frac{4}{3} \sin ^{4}\left(\frac{\theta}{2}\right)(4 \beta \cos (\theta)+2 \beta-3) \\
& +i \frac{1}{3} \sin (\theta)[2 \beta \cos (2 \theta)-3(2 \beta+1) \cos (\theta)+4 \beta+6]
\end{aligned}
$$

\footnotetext{
${ }^{\S}$ Nyukhtikov et al. ${ }^{24}$ presented a slightly different exercise when deriving their optimized second-order scheme, known as BDF2OPT( $\left.\beta=0.58\right)$. Their primary focus was the accuracy of four-level schemes, and did not specifically require $A$-stability.
} 
and $A$-stability is guaranteed if $\operatorname{Re}(\mu) \geq 0$ for all $\theta$. Differentiating $\operatorname{Re}(\mu)$ with respect to $\theta$ in equation 3 and solving for all roots reveals that extrema exist at

$$
\{x \rightarrow n \pi ; n=0, \pm 1, \pm 2, \cdots\},\left\{x \rightarrow \pm 2 \cos ^{-1}\left( \pm \frac{\sqrt{2 \beta+1}}{2 \sqrt{\beta}}\right)\right\}
$$

The values of $\operatorname{Re}(\mu)$ at these extrema are

$$
\left\{0, \frac{8 \beta}{3}+4,-\frac{(2 \beta-1)^{3}}{12 \beta^{2}}\right\}
$$

If all the extremum values are strictly non-negative, then the resulting scheme is $A$-stable. Thus, the $\operatorname{BDF} 2 \mathrm{OPT}(\beta)$ family of schemes is $A$-stable for $\beta$ on the interval $0 \leq \beta \leq \frac{1}{2}$.

Note that among the four-time-level $A$-stable $\operatorname{BDF} 2 \mathrm{OPT}(\beta)$ schemes, the most accurate is achieved for the value $\beta=\frac{1}{2}$, which will be referred to as BDF2OPT(4). For this case, the leading order truncation term is exactly half of the original BDF2 scheme: i.e. $C=\frac{-2}{12}$. It is also noted that the BDF2OPT scheme of Nyukhtikov et al. ${ }^{24}$ which was constructed using $\beta=0.58$, is $A(\alpha)$-stable, with $\alpha=89.89^{\circ}$, and hence is weakly unstable.

The stability characteristics of the four-time-level $\operatorname{BDF} 2 \mathrm{OPT}(\beta)$ family of schemes for two $\beta$ parameters are listed in Table 2.

\begin{tabular}{|c|c|c|c|}
\hline Method & Levels & $\beta$ & $A(\alpha)$ \\
\hline BDF2OPT(4) & 4 & 0.50 & $A\left(90.00^{\circ}\right)$ \\
BDF2OPT $(\beta=0.58)$ & 4 & 0.58 & $A\left(89.89^{\circ}\right)$ \\
\hline
\end{tabular}

Table 2. Properties of four-time-level BDF2OPT( $\beta)$ methods.

A similar exercise can be performed by parameterizing the BDF2 scheme with two additional time levels:

$$
\operatorname{BDF} 2 \mathrm{OPT}(\beta, \gamma)=\gamma \mathrm{BDF} 4+\beta \mathrm{BDF} 3+(1-\beta-\gamma) \mathrm{BDF} 2
$$

The $A$-stable scheme having the lowest error constant is achieved for the case $\gamma=1-\frac{1}{\sqrt{2}}$, and $\beta=-\frac{5}{2}+2 \sqrt{2}$ which will be referred to by BDF2OPT(5).

Fig. 1 (a) shows five stability locus curves. The inner and outermost curves correspond to BDF2 and BDF4, respectively. It is observed from this figure that a small portion of the root locus of the BDF3 and BDF4 schemes lies in the LHP, which indicates the $A(\alpha)$-stable nature of these schemes. The BDF2OPT(4) and BDF2OPT(5) schemes do not cross into the LHP, and are therefore indicative of an $A$-stable scheme, just as is the case for the BDF2 scheme. Fig. 1 (b) shows an expanded view of the stability locus near the origin. Observe that except for the origin, the BDF2OPT(4) scheme is bounded away from the imaginary axis while the BDF2OPT(5) scheme touches the axis at precisely two additional points.

This stability analysis is further substantiated with a numerical test. The model equation $U_{t}+U_{x}=0 ; 0 \leq x \leq$ $1, t \geq 0$ is integrated in time using the $\operatorname{BDF} 2 \mathrm{OPT}(\beta)$ schemes for $\beta=0(B D F 2), 0.45,0.48,0.50,0.58,1.0(B D F 3)$ as well as the five level BDF2OPT(5) scheme. The initial condition is a $U(x, 0)=\sin (2 \pi x)$. The spatial derivative $U_{x}$ is discretized using in all cases a skew-symmetric, second-order, central-difference operator. The skew-symmetry of the matrix is maintained near the boundaries by adopting a periodic spatial domain, thereby guaranteeing that the semidiscrete eigenvalues are confined to the imaginary axis. This setting increases the probability that at least one eigenvalue will reside in the unstable lobes of the $\operatorname{BDF} 2 \mathrm{OPT}(\beta)$ schemes for $\beta>\frac{1}{2}$. Fig. 2 shows a convergence study comparing the aforementioned $\operatorname{BDF} 2 \mathrm{OPT}(\beta)$ schemes. The solution error is plotted as a function of the time-step $\left(\mathrm{CFL}=\frac{\Delta t}{\Delta x}\right.$ with $\delta x$ fixed and $\delta t$ varying) on a logarithmic plot. All schemes converge over at least a portion of the time-step refinement, and the convergence rate is design order in all cases. The cases for which the parameter $\beta$ exceeds the threshold $\beta=\frac{1}{2}$ are only conditionally stable, as is indicated by the sudden divergence of the solutions. It is also noted from Fig. 2, that the formal accuracy of $\mathrm{BDF} 2$ and $\operatorname{BDF} 2 \mathrm{OPT}(\beta)$ schemes are the same (i.e. second order), whereas the actual error in the solutions for the BDF2OPT(4) scheme is approximately half compared to the BDF2 solutions at a given time step.

A question arises concerning the efficacy of the BDF scheme derived from the five-level case: BDF2OPT(5). In this case, the stability locus curve touches the imaginary axis at two points in addition to the origin. Modes that coincide with these two points on the imaginary axis will be neutrally stable and could potentially lead to stability problems in the presence of algebraic perturbations resulting from inadequate convergence of the nonlinear system at each time-level. The stability characteristics of the BDF2OPT(5) scheme remains the ongoing focus of investigation, as does the task of finding the general expression of the coefficients for an arbitrary number of time levels. 
In summary, the BDF2 scheme has been re-optimized using slightly different criteria than those proposed by Nyukhtikov et al. ${ }^{24} \mathrm{It}$ is proven that the leading-order truncation error of the conventional BDF2 scheme can be decreased by a factor 2 or $\frac{2}{5-3 \sqrt{2}} \approx 2.64$ by combining it with the BDF3 and BDF3/BDF4 schemes, respectively.

\section{Results}

For practical 3-D aerodynamic applications, it is important to select a temporal scheme that is efficient and robust, and does not adversely impact the performance of the base solver. Having examined various higher order temporal schemes, it was decided to use the BDF2OPT scheme with four time levels described by Eqn. (1) in the previous section. This scheme requires the solution vector be stored at one additional time level compared with the standard BDF2 scheme; however, the lead truncation error term is nearly halved with only a nominal increase in the operation count.

We now examine the turbulent flow past a circular cylinder at a free stream Mach number of 0.166 and at a Reynolds number of 166,000 based on cylinder diameter, D, to match the test conditions of the experimental study of Jenkins et al. ${ }^{25}$ The turbulence model used for this case is based on the two-equation shear stress transport (SST) model of Menter. ${ }^{14}$ It is well known that turbulence models developed for steady flows, such as the SA and SST models, are overly diffusive ${ }^{26,27}$ and suppress the three-dimensionality of the flow in CFD simulations for unsteady flows that are accompanied by large reverse flow regions. The Detached Eddy Simulation (DES) model suggested by Spalart ${ }^{28}$ has been used with reasonable success for the computation of unsteady flows past circular cylinders. ${ }^{26,29-31}$ Others have used various forms of hybrid RANS/LES models for such computations. ${ }^{32,33}$ In the present work, the SST model was modified such that the production terms were set to zero outside the boundary-layer region in the manner described by Khorrami et al. ${ }^{27}$ and Lockard et al. ${ }^{34}$ which results in a much less diffusive model and lower eddy viscosity levels in the wake regions.

Wall temperature based on adiabatic flat plate conditions ${ }^{11}$ is imposed on the surface of the cylinder along with the no slip conditions. Riemann-invariant based boundary conditions are imposed on the far-field boundary. In the spanwise direction, periodic boundary conditions are imposed at $z=0$ and $z=z_{\max }$ to more closely simulate an infinite span problem. For the initial computations, the far-field boundary is set at a distance of 15 cylinder diameters away from the center of the circular domain and a span of 3 cylinder diameters is used. A structured grid consisting of 289 x 289 points in the circumferential and radial direction was created in a two-dimensional plane, and extruded to produce 97 equally spaced planes in the spanwise direction to create the three-dimensional grid used in these computations. The grid spacing was clustered near the cylinder surface with a normal grid spacing equal to 0.0002 of a cylinder diameter at the surface which results in a value the turbulent parameter, $y^{+}$of order 1. A partial view of the grid in the end plane is shown in Fig. 3, where the grid clustering near the surface and wake region is clearly visible. The choice of grid clustering in the wake region was influenced by the Detached Eddy Simulation (DES) arguments proposed by Spalart ${ }^{28}$ and to replicate the grid topology suggested for DES simulations for cylinders by Travin et al. ${ }^{26}$ and Vatsa and Singer. ${ }^{31}$

\section{IV.A. Original $\operatorname{BDF2OPT}(\beta=0.58)$ scheme}

The first series of results reported here were obtained with the BDF2OPT $(\beta=0.58)$ scheme originally proposed by Nyukhtikov et al. ${ }^{24}$ for improving the accuracy of BDF2 scheme. Although this scheme is $A(\alpha)$-stable and, therefore, conditionally stable, its stability range is much broader than the BDF3 scheme (see Tables 1-2), and it is more accurate than the conventional BDF2 scheme at nominally the same computational cost. This scheme was used originally by Nyukhtikov et al. ${ }^{24}$ for several turbomachinery problems and did not show any signs of instability. Vatsa and Carpenter ${ }^{23}$ have examined the performance of this scheme for the unsteady Navier-Stokes equations and demonstrated the improved accuracy of BDF2OPT scheme compared to the BDF2 scheme for several test problems without encountering any numerical instability. The BDF2OPT scheme has also been used successfully with the FUN3D code for several problems of aerodynamic interest by Biedron et al. ${ }^{35,36}$ In the present work, we undertook the task of assessing the performance and accuracy of this scheme along with that of the standard BDF2 scheme for computing the unsteady flow past a circular cylinder over long periods of time with eventual application to acoustic noise prediction.

The flow field was initialized as free stream and the FUN3D code was run for about 2000 iterations in a steady mode using the standard SST turbulence model. These solutions were specified as initial conditions for the unsteady flow computations performed with the modified SST turbulence model described earlier in the previous section. As can be seen from Fig. 4, care was taken to ensure that the residuals in the dual time-stepping converge 3-4 orders for the hydrodynamic equations. The values in the plot are the $\mathrm{L}_{2}$ norm of the subiteration residuals over the entire computational domain. The residuals for the turbulence equations only drop 2 orders of magnitude because the convergence is poor in highly unsteady regions away from the body where the modifications to the SST model minimizes the influence of the turbulence model. 
Instantaneous contour plots of the density in the $\mathrm{z}=0$ plane, based on flow solutions obtained after running $10 \mathrm{~K}, 20 \mathrm{~K}$ and $26 \mathrm{~K}$ time steps (K represents a value of 1000 in this context, thus $10 \mathrm{~K}$ stands for 10,000$)$ with this $\mathrm{BDF} 2 \mathrm{OPT}(\beta=$ 0.58) scheme are shown in Figs. 5(a)-(c), respectively. Fig. 5 (a) displays the typical vortex pattern behind the cylinder which is confined to the wake region and becomes weaker further away from the cylinder. The fluctuations in the flow field away from the cylinder and wake region are primarily acoustic and cannot be seen on this scale at this instant in time. However, when we examine the solutions at $20 \mathrm{~K}$ time steps, the density contours indicate the formation of spurious waves. These waves get stronger with time (see Fig. 5 (c) at $26 \mathrm{~K}$ steps), and eventually overwhelm the solution in the whole domain.

Although the analysis in section III would suggest that the $\operatorname{BDF} 2 \mathrm{OPT}(\beta=0.58)$ is admitting instabilities because it is only conditionally stable, the problem being solved here is much more complicated than the model problem used for the analysis. Hence, numerical experimentation was used to rule out other possible sources of the spurious oscillations. Several possible causes for the instabilities were investigated in a systematic fashion. Characteristic-based boundary conditions in the downstream region of the viscous wake can induce numerical instabilities, especially in subsonic flow regions when sufficiently fine grids are used near the downstream boundary. The combination of specifying the downstream pressure with several different forms of far-field characteristic conditions had minimal effect. The problem also persisted with tetrahedral grids, which are more common for unstructured grid codes.

The distance of the far-field boundary was then doubled, i.e. it was placed at a distance of 30 cylinder diameters away from the center of the cylinder. We also coarsened the grid in the far-field to be more isotropic so as to reduce the reflections from the far field. A global view of this grid in the $\mathrm{z}=0$ plane is presented in Fig. 6, where an inviscid type of grid is seen near the far-field boundary. The solutions obtained on this grid after $15 \mathrm{~K}$ and $30 \mathrm{~K}$ time steps are shown in Figs. 7 (a) and (b), respectively. Whereas the solution after $15 \mathrm{~K}$ time steps is well behaved and displays the expected trends, the instabilities have polluted the flow structure completely by the later point in time at $30 \mathrm{~K}$ time steps.

Several other numerical studies were conducted to isolate the cause. We discovered that these instabilities could be suppressed by coarsening the spanwise grid distribution by a factor of 2 . Conversely, if the spanwise extent was reduced by half, while still maintaining the number of points in the spanwise direction of the original grid, spurious oscillations were observed in the computational results after about $25 \mathrm{~K}$ time steps. Based on this exercise, we inferred that these numerical instabilities are more likely to appear for cases where the numerical dissipation is reduced via finer grids.

As a final step to ensure that there were no other sources of instabilities in our numerical scheme, the computations

were repeated with the standard BDF2 scheme, which is theoretically unconditionally stable or $A$-stable. Computational results after 50K time steps with the BDF2 scheme are shown in Figs. 8 (a)-(b), and these results display no sign of instabilities.

Based on the results obtained in these tests, the $\operatorname{BDF} 2 \mathrm{OPT}(\beta=0.58)$ scheme appears to permit certain unstable modes to grow because of its conditional stability. One may not encounter such instabilities in the initial stages of a computation, or for problems where sufficient physical or numerical dissipation is present to damp these out. However, one should exercise caution when using this scheme for problems requiring integration over long periods of time.

\section{IV.B. Modified BDF2OPT $(\beta=0.48)$ scheme}

For the next series of solutions, we used the modified $\operatorname{BDF} 2 \mathrm{OPT}(\beta)$ scheme with a value of $\beta=0.48$. Based on the stability analysis presented in Section III of this paper, it is recognized that the BDF2OPT schemes are $A$-stable for any value of $\beta \leq 0.50$. A value of 0.48 for the $\beta$ parameter was chosen to be conservative. The resulting scheme is an $A$-stable scheme, and it reduces the temporal error by approximately a factor of 2 when compared with the standard BDF2 scheme. For remainder of this paper, solutions are presented on the grid where far-field boundary was placed at a distance of $30 \mathrm{D}$ away from center (see Fig. 6).

The instantaneous density contours in the $\mathrm{z}=0$ plane after $50 \mathrm{~K}$ time steps with the modified $\mathrm{BDF} 2 \mathrm{OPT}(\beta=0.48)$ scheme are shown in Fig. 9 (a)-(b). These results are free of any sign of instability and are qualitatively similar to the results obtained with the standard BDF2 scheme shown in Figs. 8 (a)-(b) for the same grid. We have run the code to 90K iterations in time to monitor the performance of this scheme, and have not detected any sign of numerical instability or spurious behavior in these solutions.

The time-averaged pressure distributions on the cylinder surface were also examined. We started averaging the flow field data after running the code for $20 \mathrm{~K}$ time steps after the initial transients had left the computational domain. The time-averaged surface pressure coefficient distribution at each circumferential location was also averaged in the spanwise direction (assuming spanwise homogeneity) to obtain the time-averaged data as a function of azimuthal angle, $\theta$. The angle is measured from the upstream stagnation point and is positive in the clockwise direction. The results from the $\mathrm{BDF} 2$ and $\operatorname{BDF} 2 \mathrm{OPT}(\beta=0.48)$ schemes are compared with experimentally measured data ${ }^{25,37}$ for the cylinder in Fig. 10. Note that the flow was tripped using a transition strip between $\theta=50-60^{\circ}$ in the experimental case to simulate 
turbulent transition on the cylinder surface, whereas the CFD code does not have the mechanism to force transition in this manner. Therefore, the CFD code was run in a fully turbulent mode. However, the actual transition in the CFD solutions occurred later compared to the transition trip location in the experiment. However, separation did occur before $\theta=90^{\circ}$ so that the flow became turbulent before separation.

The pressure distributions from the two temporal schemes in FUN3D are almost indistinguishable in Fig. 10. The computational results compare well with the measured data near the stagnation region as well as on the aft side of the cylinder. However, the computational $C_{p}$ indicates an earlier separation, thereby resulting in under-expansion of the flow near the top and bottom of the cylinder. We believe that at this moderate value of Reynolds number, the CFD code with the SST turbulence model does not simulate the same transition to turbulent flow as in the experiment. Hence, the discrepancy in the pressure comparisons. We also obtained solutions for this case on the same grid with the structured grid flow solver CFL3D ${ }^{38,39}$ using the BDF2 scheme, and the resulting pressure distributions from these computations are also shown in Fig. 10. The pressure distributions obtained from the CFL3D and FUN3D codes are in excellent agreement with each other.

The distribution of the root-mean-square (rms) perturbed surface pressure coefficient $\left(C p_{r m s}^{\prime}\right)$ is shown in Fig. 11. The FUN3D solutions obtained with the BDF2 and BDF2OPT schemes are compared with the experimental data obtained in the Quiet Flow Facility at NASA Langley Research Center. ${ }^{37}$ The unsteady pressure transducers on the cylinder were located every $45^{\circ}$. To obtained refined azimuthal resolution, the cylinders were rotated, and the transition strip had to be re-applied after each rotation. Such an arrangement can easily create some non-uniformity in the configuration and flow characteristics and introduce scatter in the measured data, as seen in Fig. 11. Again, the FUN3D results with the two temporal schemes compare well with each other and are in good agreement with the CFL3D results. The numerical solutions miss the peak in the rms because the flow separates too early in numerical simulations. However, most of the computed rms pressure distribution lies within the scatter of the experimental data.

Comparing the efficiency of temporal schemes in real-world settings is often a very difficult task. For example, see Ref. 9 for a thorough discussion on this topic. To actually demonstrate any accuracy differences between the time integration methods for the cylinder shedding problem, a systematic time-step refinement would be required. However, the case is dominated by fluctuations at the vortex shedding frequency. Any improvement in the time integration would only have a minor effect on the time-averaged quantities examined here unless the time step was large enough to alter the shedding process. Theoretically, one should be able to use a larger time step with the improved scheme, but exactly how much larger is difficult to estimate because of the interaction of the temporal and spatial operators as well as the complicated equation sets that are solved in real-world problems. Most CFD codes are limited by spatial rather than temporal accuracy, so the optimal time step would be difficult to determine without a systematic refinement in space and time. However, such studies are often prohibitively expensive for unsteady flow problems. The temporal and spatial resolutions chosen are often based on formulas derived from simplified equation sets and engineering judgment. Hence, an improvement in the temporal accuracy is often used like a factor of safety. In this case, one is trading a little higher operation count and the storage of one additional solution vector for the improved factor of safety.

\section{Concluding Remarks}

A new class of optimized, second-order, backward-difference (BDF2OPT) schemes has been developed that possess formal stability properties with only a slight reduction in the theoretical accuracy of previous optimized schemes. Several numerical studies were conducted to demonstrate that the $\operatorname{BDF} 2 \mathrm{OPT}(\beta=0.58)$ scheme of Nyukhtikov et al. ${ }^{24}$ does

admit spurious solutions for long-time integration of the flow over a circular cylinder. This scheme is only conditionally stable and, under certain circumstances, permits the growth of instability modes. A slight modification of the scheme results in an unconditionally stable scheme. Results obtained with the modified scheme were shown to be well behaved and free of any spurious oscillations. The proposed $\operatorname{BDF} 2 \mathrm{OPT}(\beta=0.48)$ scheme still offers improved temporal accuracy compared with the standard BDF2 scheme at nominally the same computational cost, but with a higher storage requirement (a 3 to $10 \%$ increase in the total memory requirement for typical CFD codes). Time-averaged solutions obtained for flow over an isolated cylinder were shown to be essentially identical between the BDF2 and new BDF2OPT $\beta=0.48)$ schemes. Although the new temporal scheme is more accurate, to achieve a decreased computational cost through a larger time step, the interactions between the spatial and temporal operators must be evaluated in the context of the equation set and geometry being solved. Such studies are often prohibitively expensive to perform computationally, and analysis techniques are not general enough to handle most real-world problems. Not only are higher-accuracy temporal and spatial schemes needed, but also robust, accurate, and efficient error estimates. Only with improved error estimates can the full potential of higher-accuracy methods be realized. 


\section{Acknowledgments}

The authors are deeply indebted to Dr. Steven J. Massey of NASA Langley Research Center for providing the FORTRAN utility for generating the computational grids for the circular cylinder used in this paper.

\section{References} 1987.

${ }^{1}$ Sawada, K. and Takanashi, S., “A Numerical Investigation of Wing/Nacelle Interferences of USB Configurations,” AIAA Paper 1987-0455,

${ }^{2}$ Yu, N., Kusunose, K., Chen, H., and Summerfield, D., "Flow Simulations for a Complex Airplane Configuration using Euler Equations," AIAA Paper 1987-0454, Jan. 1987.

${ }^{3}$ Jameson, A., Baker, T., and Weatherill, N., "Calculation of Inviscid Flow Over a Complete Aircraft," AIAA Paper 1986-0103, Jan. 1986.

${ }^{4}$ Reuther, J., Jameson, A., Martinelli, L., and Saunders, D., "Aerodynamic Shape Optimization of Complex Configurations via an Adjoint Formulation," AIAA Paper 1996-0094, Jan. 1996.

${ }^{5}$ Buning, P., Chiu, I. T., Obayashi, S., Rizk, Y., and Steger, J., "Numerical Simulation of Integrated Space Shuttle Vehicle in Ascent," AIAA Paper 1988-4359 CP, Aug. 1988.

${ }^{6}$ Rumsey, C., Morrison, J., and Biedron, R., "CFD Variability for a Civil Transport Aircraft Near Buffet Onset Conditions," NASA TM 2003212149, Feb. 2003.

${ }^{7}$ Kennedy, C. A. and Carpenter, M. H., "Additive Runge-Kutta Schemes for Convection-diffusion-reaction Equations," Applied Numerical Mathematics, Vol. 44, 2003, pp. 139-181.

8 van Zuijlen, A., de Boer, A., and Bijl, H., "Higher-order Time Integration Through Smooth Mesh Deformation for 3D Fluid-structure Interaction Simulations," Journal of Computational Physics, Vol. 224, No. 1, 2007, pp. 414-430.

${ }^{9}$ Carpenter, M., Kennedy, C., and Bijl, H., "Fourth-order Runge-Kutta schemes for fluid mechanics applications," Journal of Scientific Computing, Vol. 25, No. 1, 2005, pp. 157-194.

${ }^{10}$ Wang, L. and Mavriplis, D., "Implicit Solution of the Unsteady Euler Equations for High-order Accurate Discontinuous Galerkin Discretizations," Journal of Computational Physics, Vol. 225, No. 2, 2007, pp. 1994-2015.

${ }^{11}$ Anderson, W. K. and Bonhaus, D. L., "An Implicit Upwind Algorithm for Computing Turbulent Flows on Unstructured Grids," Computers and Fluids, Vol. 23, No. 1, 1994, pp. 1-21.

${ }^{12}$ FUN3D Web page: http://fun3d.larc.nasa.gov, March 2009.

${ }^{13}$ Spalart, P. and Allmaras, S., "A One-equation Turbulence Model for Aerodynamic Flows," AIAA Paper 1992-0439, Jan. 1992. 1605.

${ }^{14}$ Menter, F. R., "Two-equation Eddy-viscosity Turbulence Models for Engineering Applications,” AIAA Journal, Vol. 32, No. 8, 1994, pp. 1598-

${ }^{15}$ Roe, P. L., “Approximate Riemann Solvers, Parameter Vectors, and Difference Schemes,” Journal of Computational Physics, Vol. 43, 1981, pp. 357-372.

${ }^{16}$ Nielsen, E., Lu, J., Park, M., and Darmofal, D., "An Implicit, Exact Dual Adjoint Solution Method for Turbulent Flows on Unstructured Grids," Computers and Fluids, Vol. 33, No. 9, 2003, pp. 1131-1155.

${ }^{17}$ Jameson, A., "Time Dependent Calculations Using Multigrid, with Applications to Unsteady Flows Past Airfoils and Wings," AIAA Paper 1991-1596, 1991

${ }^{18}$ Gear, C., Numerical Initial Value Problems in Ordinary Differential Equations, Prentice Hall, 1971.

${ }^{19}$ Hairer, E., Norsett, S., and Wanner, G., Solving Ordinary Differential Equations I, Springer-Verlag, 2nd ed., 1993.

${ }^{20}$ Dahlquist, G., "A Special Stability Problem for Linear Multistep Methods," BIT Numerical Mathematics, Vol. 3, No. 1, March 1963, pp. $27-43$.

${ }^{21}$ Hairer, E. and Wanner, G., Solving Ordinary Differential Equations II, Springer-Verlag, 2nd ed., 1996.

${ }^{22}$ Melson, N., Sanetrik, M., and Atkins, H., "Time-accurate Calculations with Multigrid Acceleration," Proceedings of the Sixth Copper Mountain Conference on Multigrid Methods, 1993.

${ }^{23}$ Vatsa, V. and Carpenter, M., "Higher Order Temporal Schemes with Error Controllers for Unsteady Navier-Stokes Equations," AIAA Paper 2005-5245, June 2005.

${ }^{24}$ Nyukhtikov, M., Smelova, N., Mitchell, B., and Holmes, D., "Optimized Dual-time Stepping Technique for Time-Accurate Navier-Stokes Calculations," Proceedings of the 10th International Symposium on Unsteady Aerodynamics, Aeroacoustics and Aeroelasticity of Turbomachines, 2003.

${ }^{25}$ Jenkins, L. N., Neuhart, D. H., McGinley, C. B., Choudhari, M. M., and Khorrami, M. R., "Measurements of Unsteady Wake Interference Between Tandem Cylinders," AIAA Paper 2006-3202, June 2006.

${ }^{26}$ Travin, A., Shur, M. L., Strelets, M., and Spalart, P. R., "Detached-Eddy Simulations Past a Circular Cylinder," Flow, Turbulence and Combustion, Vol. 63, 1999, pp. 293-313.

${ }^{27}$ Khorrami, M. R., Singer, B. A., and Berkman, M. E., "Time-accurate Simulations and Acoustic Analysis of Slat Free Shear Layer," AIAA Journal, Vol. 40, No. 7, 2002, pp. 1284-1291.

${ }^{28}$ Spalart, P. R., "Young Person's Guide to Detached-Eddy Simulation Grids,” NASA CR-211032, 2001.

${ }^{29}$ Strelets, M., "Detached Eddy Simulation of Massively Separated Flows," AIAA Paper 2001-0879, 2001.

${ }^{30}$ Shur, M. L., Spalart, P. R., Strelets, M., and Travin, A. K., "Modification of S-A subgrid model in DES aimed to prevent activation of the low-Re terms in LES mode," Proceedings of workshop on DES, St. Petersburg, July 2-3, 2003.

${ }^{31}$ Vatsa, V. N. and Singer, B., "Evaluation of a Second-Order Accurate Navier-Stokes Code for Detached Eddy Simulation Past a Circular Cylinder," AIAA Paper 2003-4085, June 2003.

${ }^{32}$ Elmiligui, A., Abdol-Hamid, K., Massey, S., and Pao, S., "Numerical Study of Flow Past a Circular Cylinder Using RANS, Hybrid RANS/LES and PANS Formulations," AIAA Paper 2004-4959, August 2004.

${ }^{33}$ Lynch, C. and Smith, M. J., "Hybrid RANS-LES Turbulence Models on Unstructured Grids," AIAA Paper 2008-3854, 2008. 
${ }^{34}$ Lockard, D. P., Khorrami, M. R., Choudhari, M., Hutcheson, F., Brooks, T., and Stead, D., “Tandem Cylinder Noise Predictions," AIAA Paper 2007-3450, 2007.

${ }^{35}$ Biedron, R., Vatsa, V., and Atkins, H., "Simulation of Unsteady Flows using an Unstructured Navier-Stokes Solver for Moving and Stationary Grids," AIAA Paper 2005-5093, June 2005.

${ }^{36}$ Biedron, R. and Lee-Rausch, E., "Rotor Airloads Prediction Using Unstructured Meshes and Loose CFD/CSD Coupling," AIAA Paper 20087341, August 2008

${ }^{37}$ Hutcheson, F. V. and Brooks, T., "Noise Radiation from Single and Multiple Rod Configurations," AIAA Paper 2006-2629, 2006.

${ }^{38}$ Rumsey, C., Biedron, R., and Thomas, J., "CFL3D: Its History and Some Recent Applications,” TM 112861, NASA, May 1997, presented at the Godonov's Method for Gas Dynamics Symposium, Ann Arbor, MI.

${ }^{39}$ Krist, S. L., Biedron, R. T., and Rumsey, C., CFL3D User's Manual (Version 5), NASA Langley Research Center: Aerodynamic and Acoustic Methods Branch, 1997. 


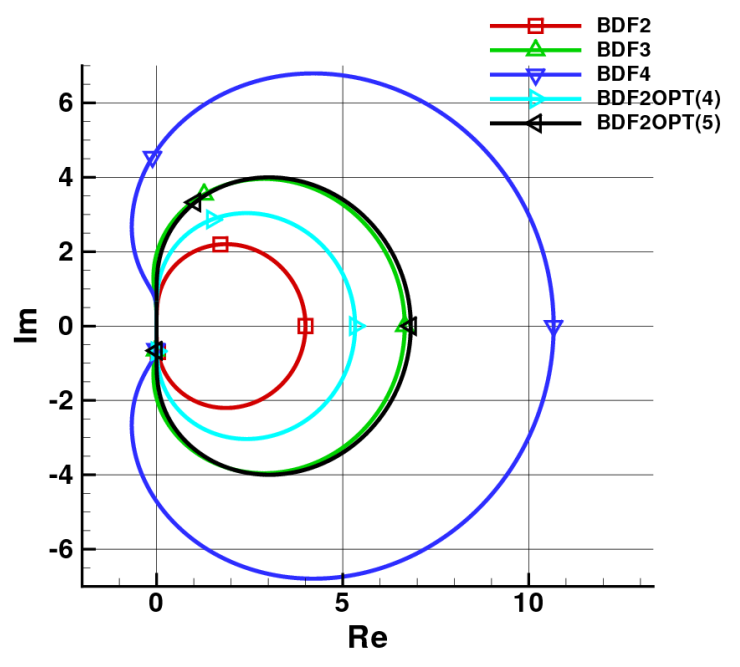

(a) Full domain view

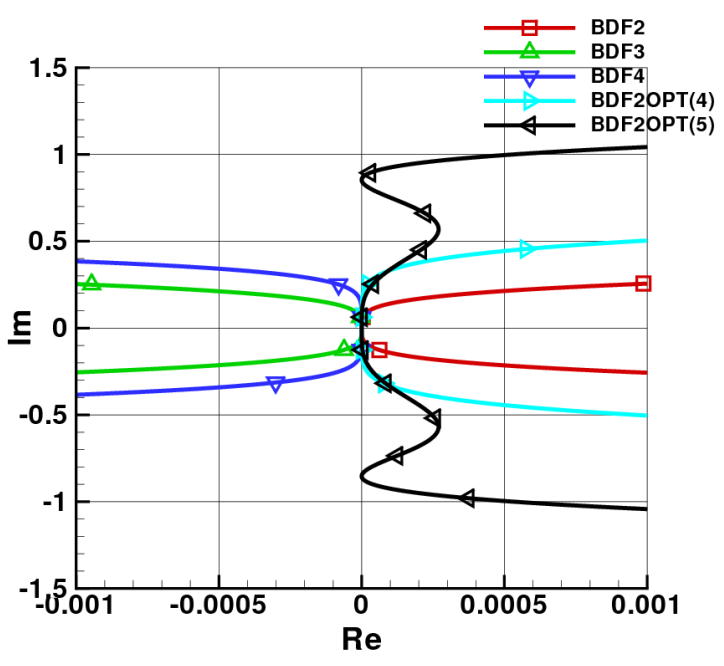

(b) Expanded view near origin

Figure 1. Stability locus for the BDF2 schemes

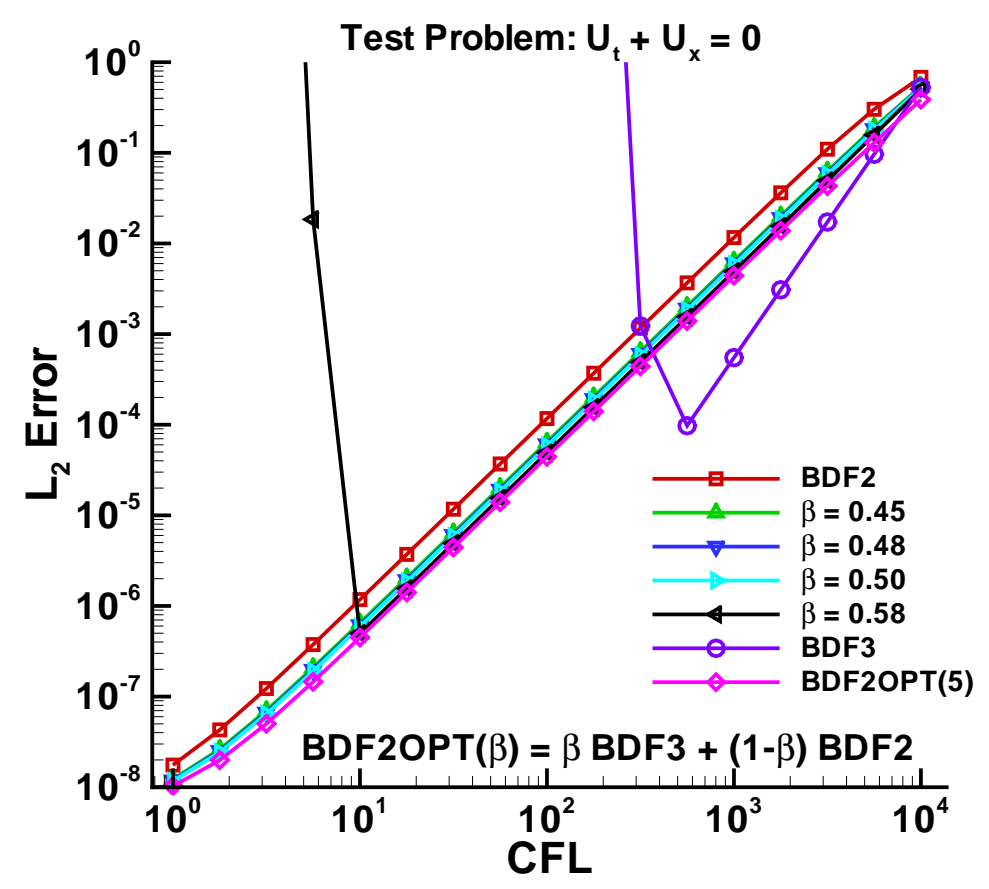

Figure 2. Convergence behavior of BDF2, BDF3 and BDF2OPT schemes for test problem 


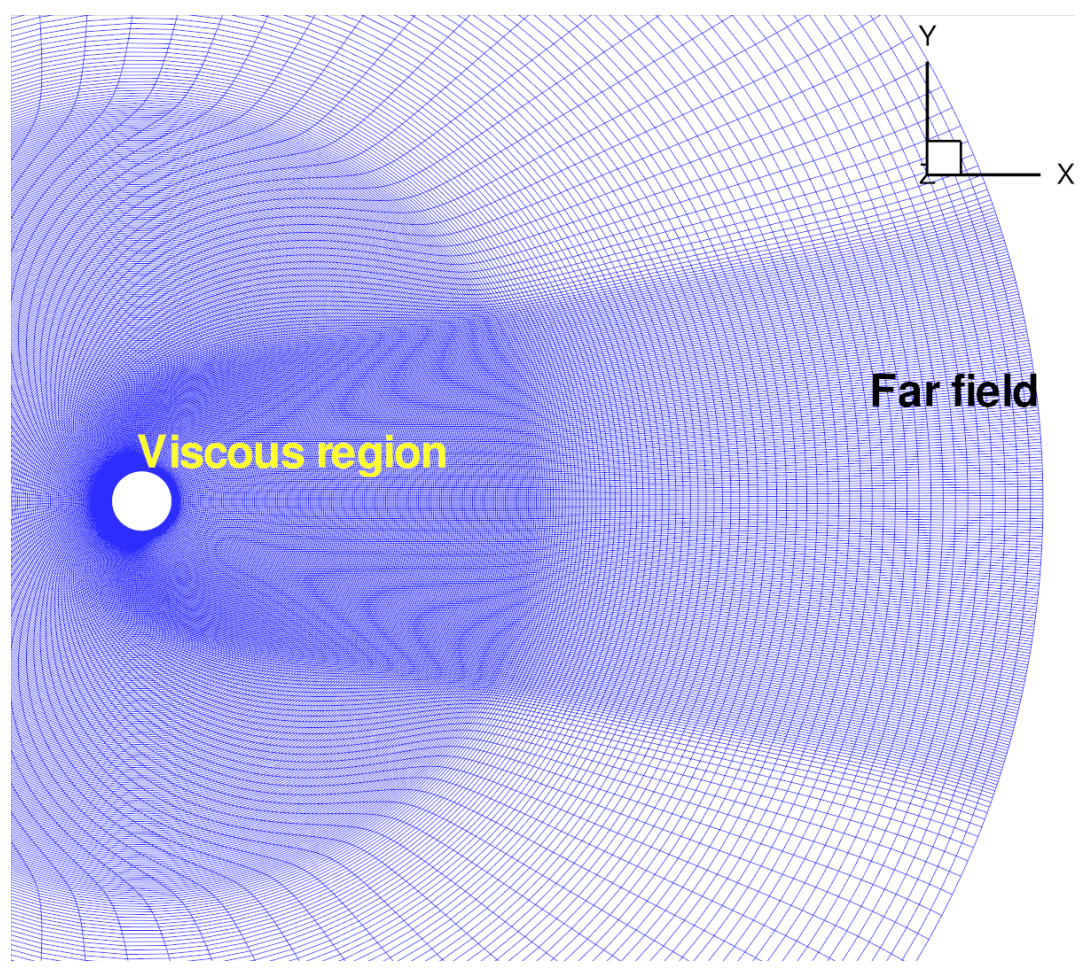

Figure 3. Partial view of grid in $\mathrm{z}=0$ plane

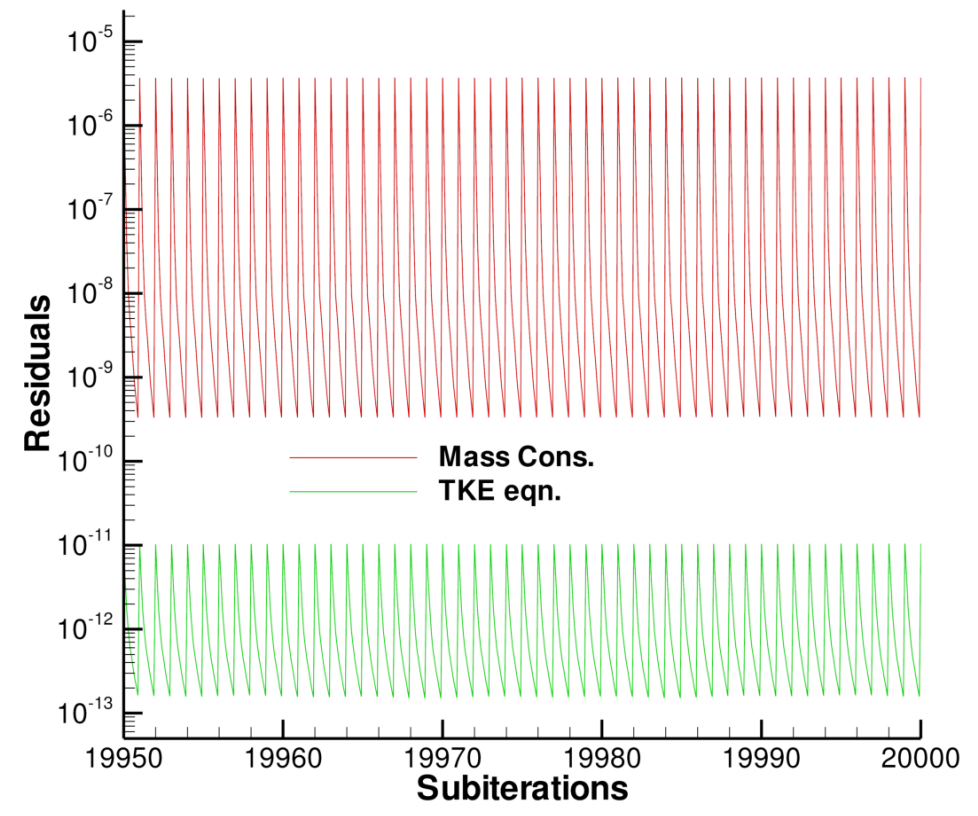

Figure 4. Convergence histories for dual-time stepping scheme 


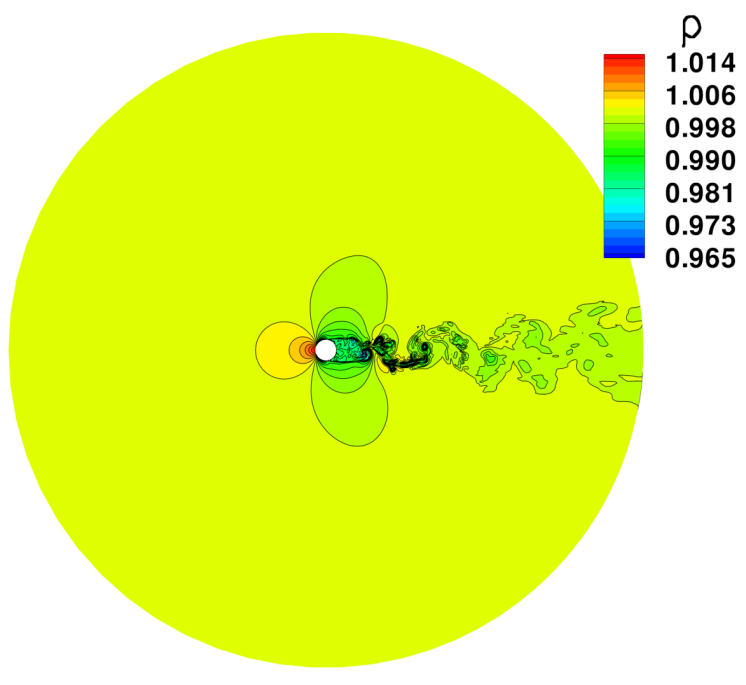

(a) $10 \mathrm{~K}$ time steps

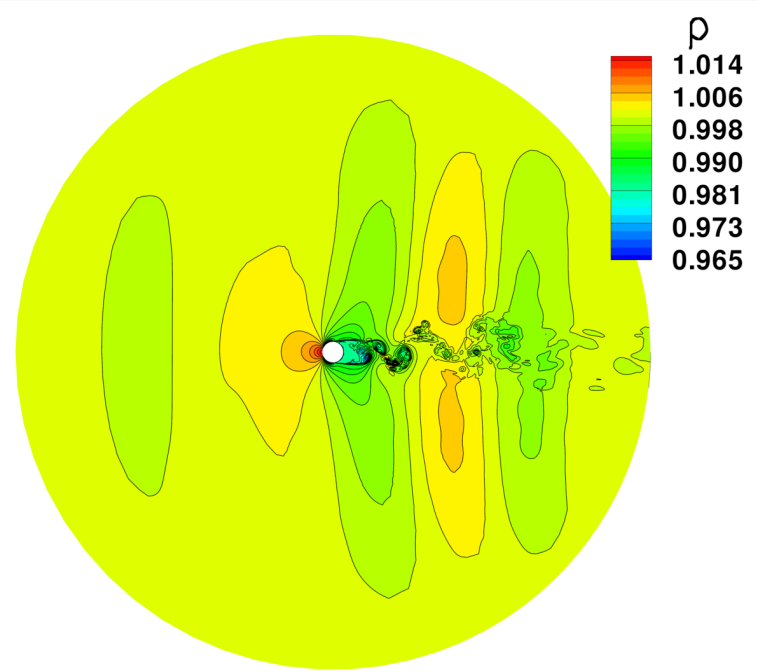

(b) 20K time steps

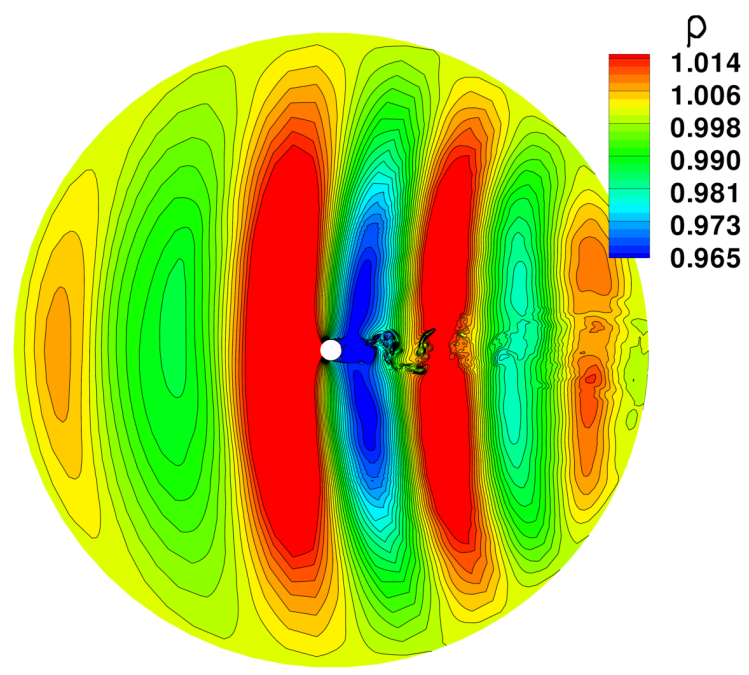

(c) $26 \mathrm{~K}$ time steps

Figure 5. Instantaneous density contours in $\mathrm{z}=0$ plane at different times. Solutions obtained using the $\operatorname{BDF} 2 \mathrm{OPT}(\beta=0.58) \operatorname{scheme}$ 


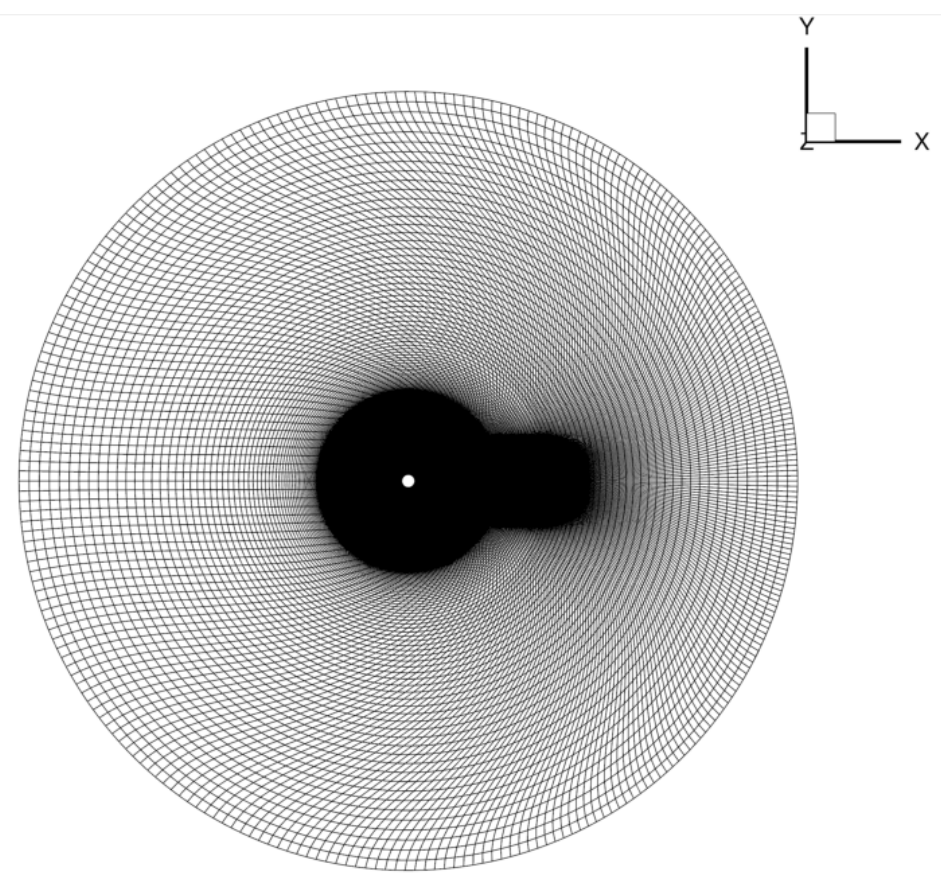

Figure 6. Global view of grid with far-field boundary at $15 \mathrm{D}$ in $\mathrm{z}=\mathbf{0}$ plane

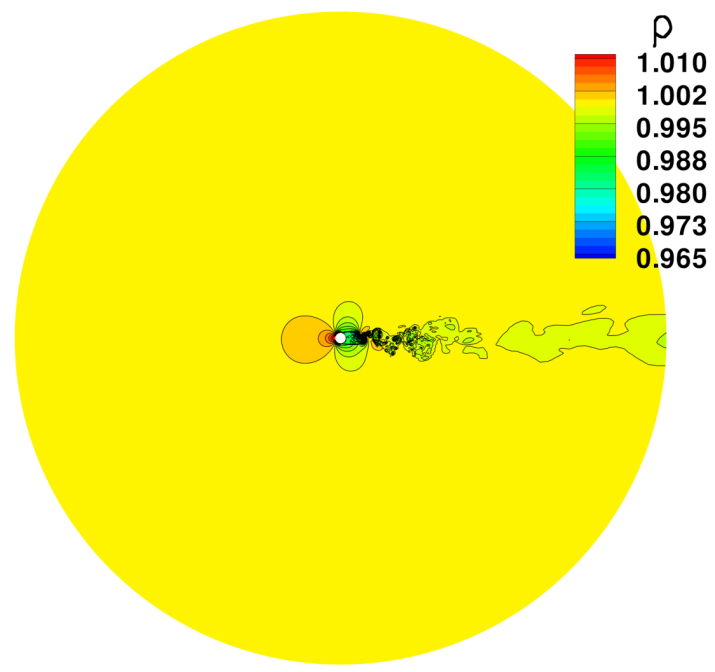

(a) $15 \mathrm{~K}$ time steps

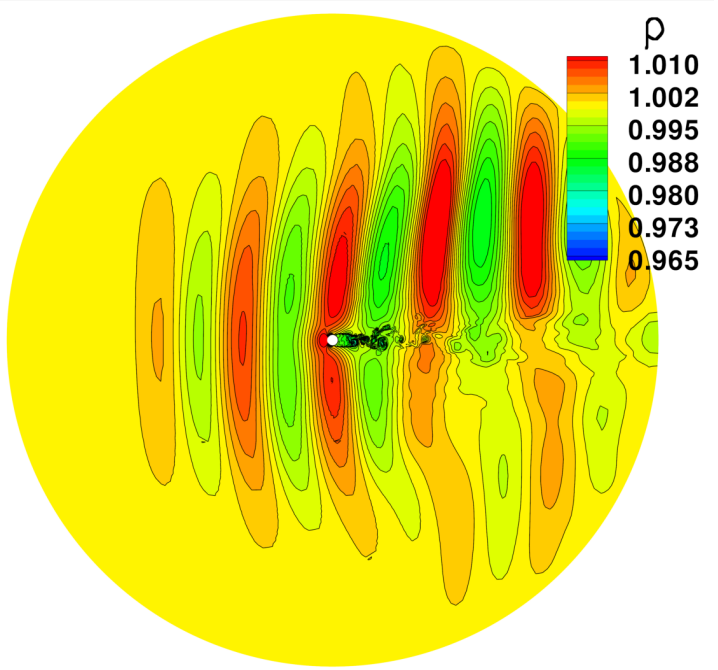

(b) $30 \mathrm{~K}$ time steps

Figure 7. Instantaneous density contours at different times, far-field boundary at 30D. Solutions obtained using the BDF2OPT $(\beta=0.58)$ scheme 


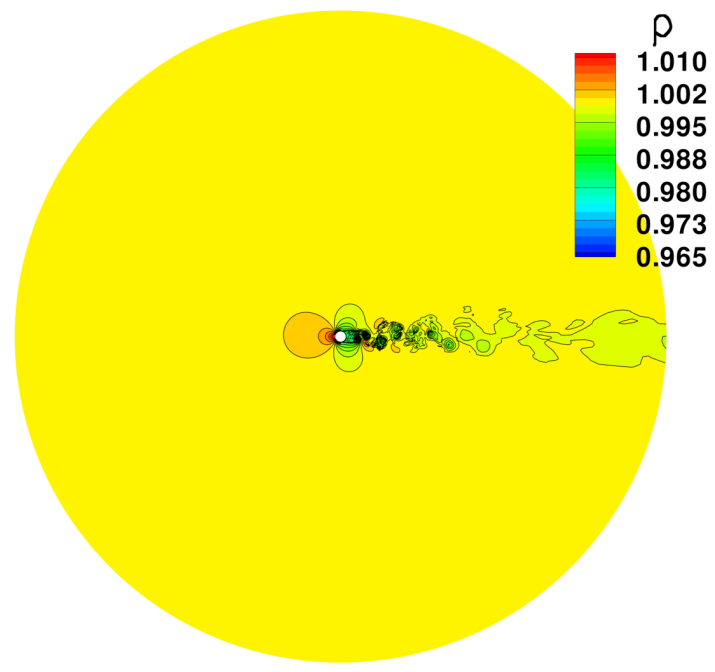

(a) Global view

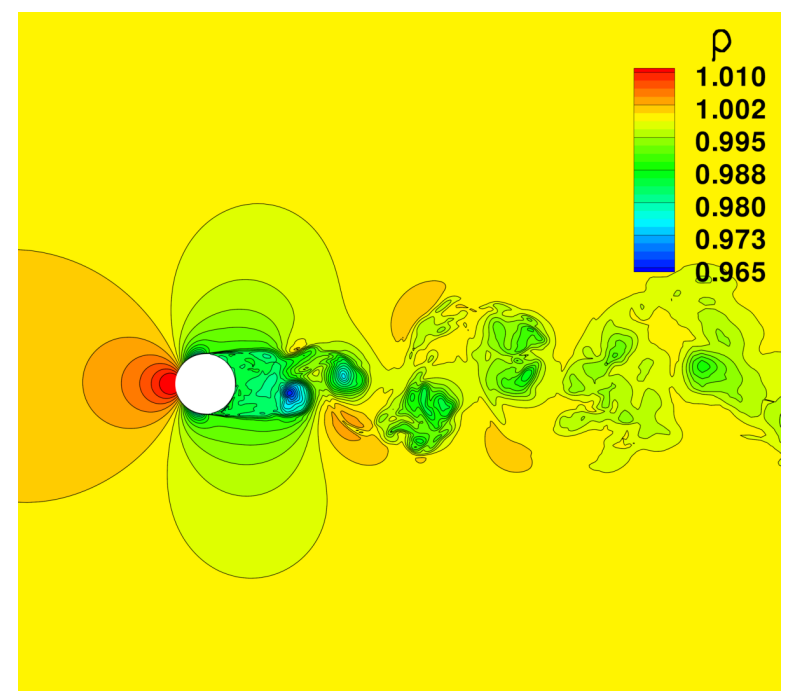

(b) Expanded view near origin

Figure 8. Instantaneous density contours at 50K time steps, far-field boundary at 30D. Solutions obtained using the BDF2 scheme

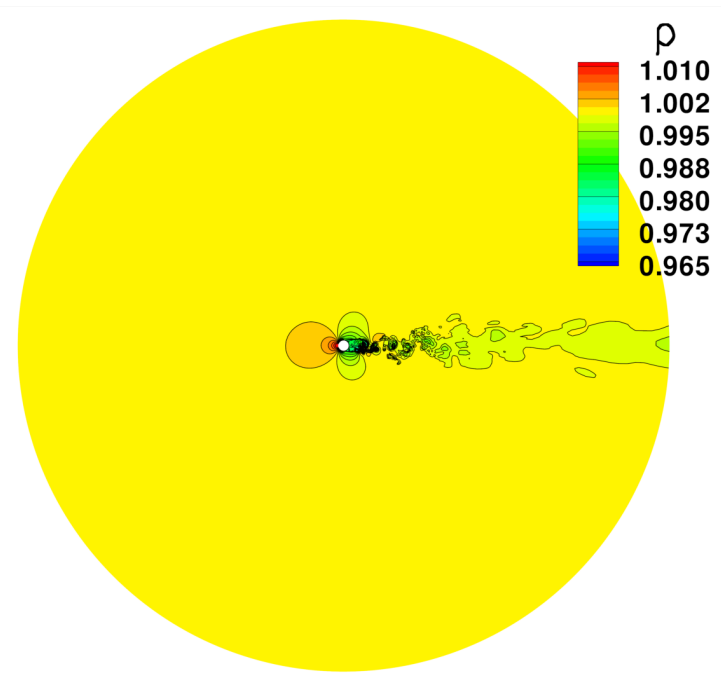

(a) Global view

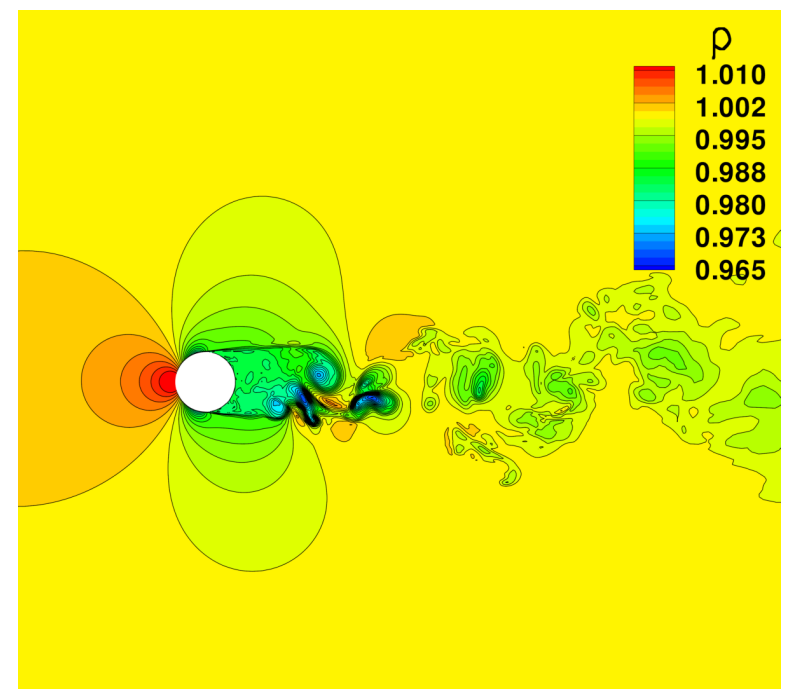

(b) Expanded view near origin

Figure 9. Instantaneous density contours at 50K time steps, far-field boundary at 30D. Solutions obtained using the BDF2OPT $(\beta=0.48)$ scheme 


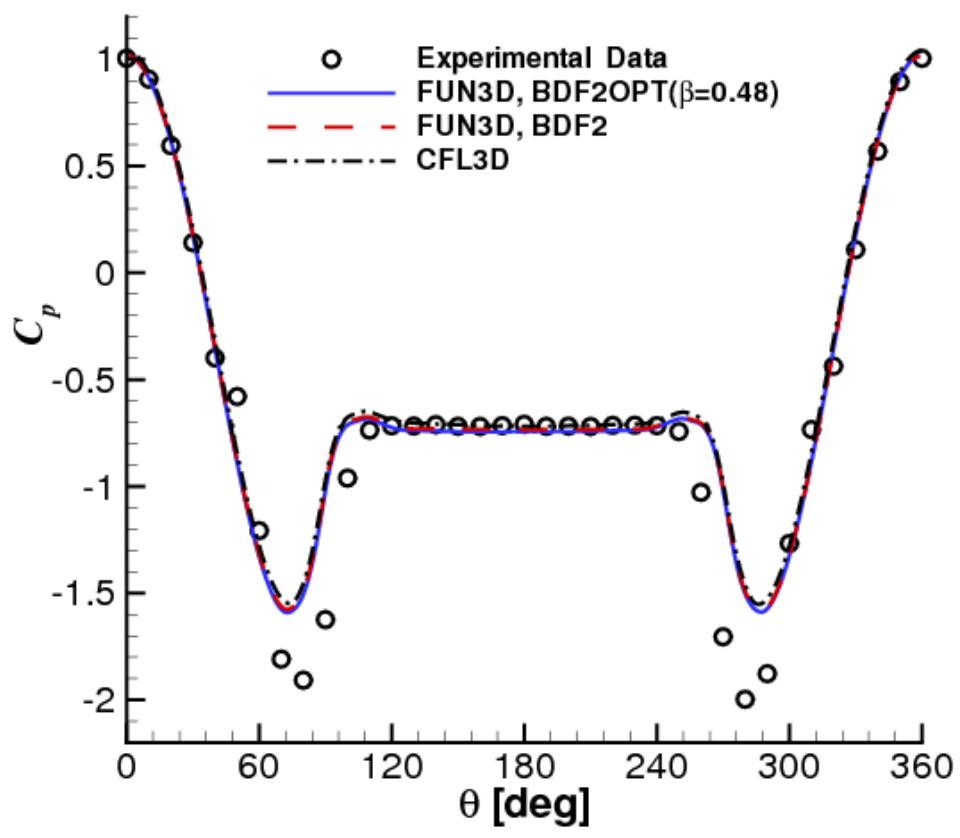

Figure 10. Comparison of time-averaged surface pressure

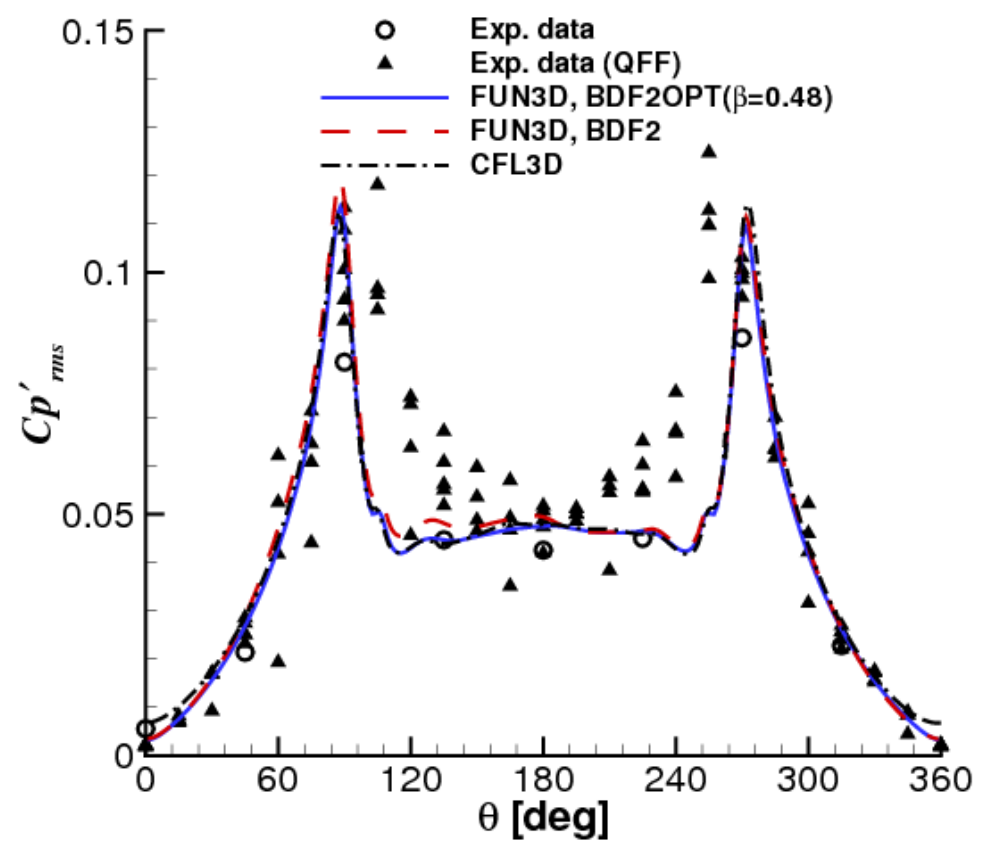

Figure 11. Comparison of perturbation pressure coefficient 